\title{
Image Processing by Three-Layer Cellular Neural Networks with a New Layer Arrangement
}

\author{
Muhammad Izzat bin Mohd Idrus, Yoshihiro Kato, \\ Yoko Uwate and Yoshifumi Nishio \\ Dept. Electrical and Electronic Eng., \\ Tokushima University \\ 2-1 Minami-Josanjima, Tokushima, Japan \\ Email: izzat, kkato, uwate, nishio@ee.tokushima-u.ac.jp
}

\begin{abstract}
In this study, we research a new layer arrangement of three layer cellular neural network (CNN). In this paper, we investigate the output characteristics by using our proposed method to image processing of gray scale image and binary image and show its effectiveness with simulation results.
\end{abstract}

\section{INTRODUCTION}

Cellular Neural Networks (CNN) were introduced by Chua and Yang in 1988 [1][2]. The idea of the CNN was inspired from the architecture of the cellular automata and the neural networks. The CNN has local connectivity property and they have been successfully developed in various image processing applications. Compare to the conventional neural networks, the CNN has been researched in various ways such as image processing and pattern recognition. In previous study of CNN, single-layer $\mathrm{CNN}$ have been introduced with many types of templates and after that, two-layer $\mathrm{CNN}$ have been proposed for high performance processing [3]. Recently, to obtain high performance in color image processing, three-layer CNN have been proposed [4].

In this study, we are using a new layer arrangement of threelayer CNN by using three types of templates. We investigate the output characteristics between the conventional CNN, twolayer CNN and the proposed CNN by apply to the binary image and gray-scale image to remove noise and edge detection simultaneously. In this paper, we describe about conventional $\mathrm{CNN}$, two-layer CNN, three-layer CNN and proposed CNN in Sec. II. In Sec. III, we show simulation results of conventional $\mathrm{CNN}$, two-layer $\mathrm{CNN}$ and proposed CNN respectively. In Sec. III also we compare between all types of structure CNN. Finally, we conclude our study in Sec. IV.

\section{Development of The Structure CNN}

In this section, we explain about the development and the characteristics of the structure CNNs which are conventional $\mathrm{CNN}$, two-layer $\mathrm{CNN}$, three-layer $\mathrm{CNN}$ and our proposed structure.

\section{A. Conventional CNN}

Conventional CNN is the first CNN proposed in [1] and only had single layer. This means that the conventional CNN has only one type of process can be done in one process. For some image processing, two or more processes have to be done to obtain the result.

\section{B. Two-layer CNN}

To obtain better result in some image processing, two-layer $\mathrm{CNN}$ has been introduced [3]. Two-layer CNN is combination of two single-layer CNNs. The two single-layer CNNs are connected by using coupling templates $C_{1}$ and $C_{2}$ which are used to connect and transferred the data between first and second layers. Compare to the conventional $\mathrm{CNN}$, two-layer CNN can be used two or more templates in one process. In our previous study [3], the two-layer $\mathrm{CNN}$ has been confirmed to have more efficient than single-layer $\mathrm{CNN}$ for some image processing.

\section{Three-layer $C N N$}

To overcome and obtain better results in color image processing, three-layer CNN have been proposed in our previous study [4]. There also have been several types of three-layer CNN. Like the two-layer CNN, to transfer between every single layers, three single CNNs are connected by using three coupling templates $C_{1}, C_{2}$ and $C_{3}$. In our previous study [4], three-layer $\mathrm{CNN}$ have been confirmed to have efficient results in color image processing.

\section{Proposed CNN}

In this study, we introduce a new structure of the CNN. The block diagram of the proposed CNN is shown in Fig .1.

Figure 1 shows that the output of the first layer CNN (CNN1) is delivered into the second layer CNN (CNN2) and the third layer CNN (CNN3) by through coupling template $\left(C_{1}\right)$. In other way, the outputs of the CNN2 and the CNN3 are delivered into the CNN1 by through coupling templates $\left(C_{2}\right)$ and $\left(C_{3}\right)$, respectively. In the previous study of threelayer $\mathrm{CNN}$ [3], all three single-layer $\mathrm{CNN}_{\mathrm{s}}$ have mutual 


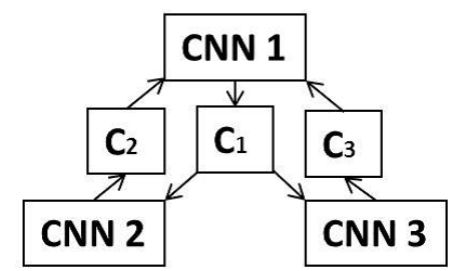

Fig. 1. Block Diagram of the proposed CNN.

connection between them. However, in our proposed CNN, CNN2 and CNN3 have not directly interacted between each layer. This arrangement is the different point between the previous structure of three-layer $\mathrm{CNN}$ and the proposed CNN.

The equations of the proposed $\mathrm{CNN}$ are described as below: (1) State equations of the proposed $\mathrm{CNN}$ :

State equation of $\mathrm{CNN1}$ :

$$
\begin{aligned}
C \frac{d v_{x 1 i j}}{d t}= & -\frac{1}{R} v_{x 1 i j}+\sum_{k=i-r}^{i+r} \sum_{l=j-r}^{j+r} A_{1}(i, j ; k, l) v_{y 1 k l}(t) \\
& +\sum_{k=i-r}^{i+r} \sum_{l=j-r}^{j+r} B_{1}(i, j ; k, l) v_{u 1 k l}(t) \\
& +\sum_{k=i-r}^{i+r} \sum_{l=j-r}^{j+r} C_{2}(i, j ; k, l) v_{y 2 k l}(t) \\
& +\sum_{k=i-r}^{i+r} \sum_{l=j-r}^{j+r} C_{3}(i, j ; k, l) v_{y 3 k l}(t)+I_{1} \\
&
\end{aligned}
$$

State equation of CNN2 :

$$
\begin{aligned}
C \frac{d v_{x 2 i j}}{d t}= & -\frac{1}{R} v_{x 2 i j}+\sum_{k=i-r}^{i+r} \sum_{l=j-r}^{j+r} A_{2}(i, j ; k, l) v_{y 2 k l}(t) \\
& +\sum_{k=i-r}^{i+r} \sum_{l=j-r}^{j+r} B_{2}(i, j ; k, l) v_{u 2 k l}(t) \\
& +\sum_{k=i-r}^{i+r} \sum_{\substack{l=j-r \\
(|i-k| \leq 1,}}^{j+r} C_{1}(i, j ; k, l) v_{y 1 k l}(t)+I_{2} \\
& \quad|j-l| \leq 1) .
\end{aligned}
$$

State equation of CNN3 :

$$
\begin{aligned}
C \frac{d v_{x 3 i j}}{d t}= & -\frac{1}{R} v_{x 3 i j}+\sum_{k=i-r}^{i+r} \sum_{l=j-r}^{j+r} A_{3}(i, j ; k, l) v_{y 3 k l}(t) \\
& +\sum_{k=i-r}^{i+r} \sum_{l=j-r}^{j+r} B_{3}(i, j ; k, l) v_{u 3 k l}(t) \\
& +\sum_{k=i-r}^{i+r} \sum_{l=j-r}^{j+r} C_{1}(i, j ; k, l) v_{y 1 k l}(t)+I_{3} \\
& (|i-k| \leq 1,|j-l| \leq 1) .
\end{aligned}
$$

(2) Output equations of the proposed CNN:

Output equation of CNN1 :

$$
v_{y 1 i j}(t)=\frac{1}{2}\left(\left|v_{x 1 i j}(t)+1\right|-\left|v_{x 1 i j}(t)-1\right|\right) .
$$

Output equation of CNN2 :

$$
v_{y 2 i j}(t)=\frac{1}{2}\left(\left|v_{x 2 i j}(t)+1\right|-\left|v_{x 2 i j}(t)-1\right|\right) .
$$

Output equation of CNN3 :

$$
v_{y 3 i j}(t)=\frac{1}{2}\left(\left|v_{x 3 i j}(t)+1\right|-\left|v_{x 3 i j}(t)-1\right|\right) .
$$

\section{Simulation Results}

In this section, we show simulation results for the conventional $\mathrm{CNN}$, the two-layer $\mathrm{CNN}$ and the proposed CNN. For simulations, we use three types of templates which are "Small object remover", "Edge detection" and "Smoothing". All of these original templates can be found in [5].

"Small object remover"

$$
A_{1}=\left[\begin{array}{lll}
1 & 1 & 1 \\
1 & 2 & 1 \\
1 & 1 & 1
\end{array}\right], B_{1}=\left[\begin{array}{lll}
0 & 0 & 0 \\
0 & 0 & 0 \\
0 & 0 & 0
\end{array}\right], I_{1}=0 .
$$

"Edge detection"

$A_{1}=\left[\begin{array}{lll}0 & 0 & 0 \\ 0 & 1 & 0 \\ 0 & 0 & 0\end{array}\right], B_{1}=\left[\begin{array}{lll}-1 & -1 & -1 \\ -1 & 8 & -1 \\ -1 & -1 & -1\end{array}\right], I_{1}=-1$.

\section{"Smoothing"}

$$
A_{1}=\left[\begin{array}{lll}
0 & 1 & 0 \\
1 & 2 & 1 \\
0 & 1 & 0
\end{array}\right], B_{1}=\left[\begin{array}{lll}
0 & 0 & 0 \\
0 & 0 & 0 \\
0 & 0 & 0
\end{array}\right], I_{1}=0 .
$$

(i) For the conventional CNN, we use two types of original templates which are "Small object remover" and "Edge detection". The feature of the template "Small object remover" is delete small noises in images and the feature of the template "Edge detection" is to detect the edge of the object.

(ii) For the two-layer $\mathrm{CNN}$, the templates are designed as below. This idea was inspired from the previous study [3].

Template of CNN1 :

$$
A_{1}=\left[\begin{array}{lll}
1 & 1 & 1 \\
1 & 2 & 1 \\
1 & 1 & 1
\end{array}\right], B_{1}=\left[\begin{array}{lll}
0 & 0 & 0 \\
0 & 0 & 0 \\
0 & 0 & 0
\end{array}\right], I_{1}=0 .
$$

Template of $C N N 2$ :

$$
A_{2}=\left[\begin{array}{lll}
0 & 0 & 0 \\
0 & 1 & 0 \\
0 & 0 & 0
\end{array}\right], B_{2}=\left[\begin{array}{lll}
0 & 0 & 0 \\
0 & 0 & 0 \\
0 & 0 & 0
\end{array}\right], I_{2}=-1 .
$$

Coupling Templates :

$$
C_{1}=\left[\begin{array}{lll}
-1 & -1 & -1 \\
-1 & 8 & -1 \\
-1 & -1 & -1
\end{array}\right], C_{2}=\left[\begin{array}{lll}
0 & 0 & 0 \\
0 & 1 & 0 \\
0 & 0 & 0
\end{array}\right]
$$

(iii) For the proposed $\mathrm{CNN}$, the templates are designed as below. We add "Smoothing" in our designed templates, because was shown to be effective in two-layer CNN for edge detection. For designed coupling templates, we designed $C_{1}$ as template "Edge detection" operation. For " $C_{2}$ ", we designed as bridge to transfer data which was output from the CNN2. For " $C_{3}$ ", it is obtained by experienced. At this moment, we still cannot explain in scientifically. 


$$
\begin{aligned}
& \text { Template of CNN1 : } \\
& A_{1}=\left[\begin{array}{lll}
1 & 1 & 1 \\
1 & 2 & 1 \\
1 & 1 & 1
\end{array}\right], B_{1}=\left[\begin{array}{lll}
0 & 0 & 0 \\
0 & 0 & 0 \\
0 & 0 & 0
\end{array}\right], I_{1}=0 .
\end{aligned}
$$

Template of CNN2 :

$$
A_{2}=\left[\begin{array}{lll}
0 & 0 & 0 \\
0 & 1 & 0 \\
0 & 0 & 0
\end{array}\right], B_{2}=\left[\begin{array}{lll}
0 & 0 & 0 \\
0 & 0 & 0 \\
0 & 0 & 0
\end{array}\right], I_{2}=-1
$$

Template of $C N N 3$ :

$$
A_{3}=\left[\begin{array}{lll}
0 & 1 & 0 \\
1 & 2 & 1 \\
0 & 1 & 0
\end{array}\right], B_{3}=\left[\begin{array}{lll}
0 & 0 & 0 \\
0 & 0 & 0 \\
0 & 0 & 0
\end{array}\right], I_{3}=-0.5
$$

Coupling Templates :

$$
\begin{aligned}
C_{1} & =\left[\begin{array}{lll}
-1 & -1 & -1 \\
-1 & 8 & -1 \\
-1 & -1 & -1
\end{array}\right], C_{2}=\left[\begin{array}{lll}
0 & 0 & 0 \\
0 & 1 & 0 \\
0 & 0 & 0
\end{array}\right] \\
C_{3} & =\left[\begin{array}{lll}
0 & 0 & 0 \\
0 & -2 & 0 \\
0 & 0 & 0
\end{array}\right]
\end{aligned}
$$

Remark: In this study, we did not simulate the three-layer CNN [4] because we did not obtain good designed templates.

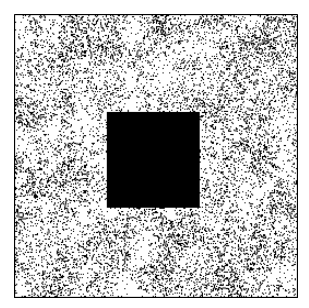

(a)

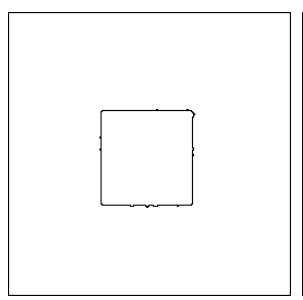

(b)

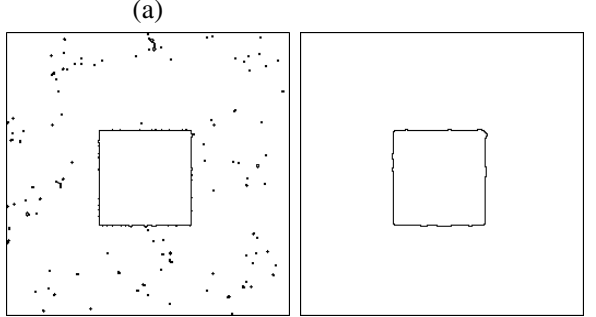

(c)

(d)
Fig. 2. Simulation results for binary images. (a) Initial image. (b) Output of the conventional CNN. (c) Output of the two-layer CNN. (d) Output of the proposed CNN.

Figure 2 shows the simulation results of binary images. Figure 2(a) shows an initial image which is a black square with noises. Figures 2(b), (c) and (d) show the outputs of the conventional $\mathrm{CNN}$, the two-layer $\mathrm{CNN}$ and the proposed CNN for binary images, respectively. In Figs. 2(b), (c) and (d), the outputs show the edge of square can be detected. In noise removal's performance, the outputs of the conventional $\mathrm{CNN}$ and proposed CNN show noises can be removed completely. However, the output of the two-layer CNN cannot remove noises completely. The results show that two-layer CNN cannot perform effectively in some image processing. From these simulation results, the conventional CNN and the proposed CNN show better results than the two-layer CNN.
However, the conventional CNN needs two processes to obtain the desired result compare to the proposed CNN. First process, we apply "Small object remover" to remove the noises. Then, for the second process, we apply "Edge detection" to detect the edge of square. From these conclusions, we can say that the proposed $\mathrm{CNN}$ exhibited excellent performance than the conventional $\mathrm{CNN}$ and the two-layer $\mathrm{CNN}$ in these simulations.

Remark: We did not use template "Smoothing" in the conventional $\mathrm{CNN}$ and the two-layer $\mathrm{CNN}$ because less effective.

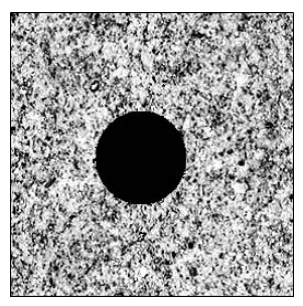

(a)

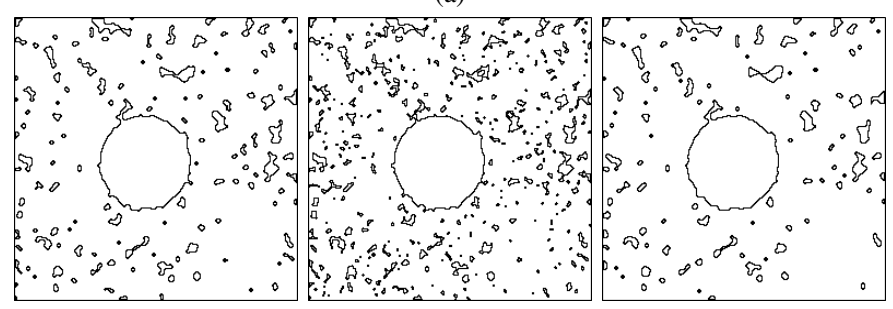

(b)

(c)

(d)

Fig. 3. Simulation results for gray-scale images. (a) Initial image. (b) Output of the conventional CNN. (c) Output of the two-layer CNN. (d) Output of the proposed CNN.

Figure 3 shows the simulation results of gray-scale images. Figure 3(a) shows an initial image which is a black circle with noises. Figures 3(b), (c) and (d) show the outputs of the conventional $\mathrm{CNN}$, the two-layer $\mathrm{CNN}$ and the proposed CNN for gray-scale images, respectively. In Figs. 3(b), (c) and (d), the outputs show that the edge of circle can be detected. In noise removal's performance, although all of the outputs cannot remove noises completely, the outputs of the conventional $\mathrm{CNN}$ and the proposed $\mathrm{CNN}$ show slightly better than the two-layer CNN. These results also show that the two-layer CNN cannot perform effectively in some image processing. For the outputs of the conventional CNN and the proposed $\mathrm{CNN}$, even the simulation results show almost the same, the output of the proposed CNN shows hardly better than the conventional CNN if we look the results in detail. Additionally, like the simulation for binary image in Fig. 2, the conventional CNN needs two processes to complete the task. For this simulation of gray-scale image, we did not say that the proposed CNN show greatly better performance than the conventional $\mathrm{CNN}$ and the two-layer CNN. However, we can say that proposed CNN shows slightly better than conventional $\mathrm{CNN}$ and two-layer CNN. 


\section{CONCLUSION}

In this study, we have proposed a new layer arrangement of three-layer CNN. In the proposed CNN, we use only one single layer $\mathrm{CNN}$ to connect with the other two single layer CNNs. From the computer simulations of simple images for binary image and gray-scale image, we investigated the output characteristics of the conventional $\mathrm{CNN}$, two-layer CNN and proposed $\mathrm{CNN}$. We can say that the proposed $\mathrm{CNN}$ have potential in image processing. However, at the moment, we do not say that our proposed CNN show excellent performance that conventional $\mathrm{CNN}$ and two-layer $\mathrm{CNN}$.

In future works, we would like to use another type of images like an image which has scenery. We also would like to use another type of templates to apply in our proposed CNN.

\section{ACKNOWLEDGMENT}

This work was partly supported by JSPS Grant-in-Aid for Scientific Research 22500203.

\section{REFERENCES}

[1] L. O. Chua and L. Yang, "Cellular Neural Networks: Theory," IEEE Trans. Circuits Syst., vol. 32, pp. 1257-1272, Oct. 1988.

[2] L. O. Chua and L. Yang, "Cellular Neural Networks: Applications," IEEE Trans. Circuits Syst., vol. 32, pp. 1257-1272, Oct. 1988.

[3] Z. Yang, Y. Nishio and A. Ushida, "Image Processing of Two-Layer CNNs:Applications and Their Stability," IEICE Trans. Fundamentals, vol. E85-A, No. 9, pp. 2052-2060, Sep. 2002.

[4] T. Inoue and Y. Nishio, "Research of Three-Layer Cellular Neural Networks Processing Color Images," IEICE Technical Report, NLP2008-25, pp. 33-36, Jul. 2008.

[5] Cellular Sensory Wave Computers Laboratory Computer and Automation Research Institute Hungarian Academy of Sciences, "Cellular wave Computing Library (Template, Algorithm, and Programs) Version 2.1" 